\title{
MEASURABLE DARBOUX FUNCTIONS
}

\author{
J. B. BROWN, P. HUMKE AND M. LACZKOVICH
}

(Communicated by R. Daniel Mauldin)

\begin{abstract}
We investigate how certain Darboux-like properties of real functions (including connectivity, almost continuity, and peripheral continuity) are related to each other within certain measurability classes (including the classes of Lebesgue measureable, Borel, and Baire-1 functions).
\end{abstract}

I. Introduction. Consider the following topological properties that functions $f$ from one topological space, $X$, to another, $Y$, may have:

Conn: $\quad f$ is a connectivity function if it is true that for every connected subset $G$ of $X, f \mid C$ is a connected subset of $X \times Y$,

D: $\quad f$ is Darboux if for every connected subset $C$ of $X, f[C]$ is a connected subset of $Y$.

For functions $f$ from the unit interval $I=[0,1]$ into the reals $R, f$ is Conn if and only if it has a connected graph. It is obvious that Conn $\Rightarrow \mathrm{D}$, but it was shown in [12] that Conn $\nLeftarrow \mathrm{D}$. On the other hand, Kuratowski and Sierpinski showed in [13] that within the class $\mathrm{B}_{1}$ of functions $f: I \rightarrow R$ of Baire's first class, Conn $\Leftrightarrow \mathrm{D}$. This result has been expanded a great deal. It is shown in Theorem 1.1, Chapter II, of Bruckner's book [3] that within $B_{1}$, the following properties are also equivalent to D:

PC: $\quad f: X \rightarrow Y$ is peripherally continuous if for each $x \in X$ and each pair of open sets $U \subseteq X$ and $V \subseteq Y$ such that $x \in U$ and $f(x) \in$ $V$, there exists an open subset $W$ of $U$, containing $x$, such that $f[\operatorname{bd}(W)] \subseteq V$. Here, bd $(W)$ denotes the boundary of $W$.

For real functions, this reduces to the condition of Young (see $[3,(2)$, p. 9]).

SM: $\quad f: I \rightarrow R$ satisfies the condition of Sen and Massera if for each $x \in I$,

$$
f(x) \in\left[\varliminf_{z \rightarrow x^{-}} f(z), \varlimsup_{z \rightarrow x^{-}} f(z)\right] \cap\left[\varliminf_{z \rightarrow x^{+}} f(z), \varlimsup_{z \rightarrow x^{+}} f(z)\right],
$$

CC: $\quad f: I \rightarrow R$ is CC if for each number $a$, the sets $\{f \leq a\}$ and $\{a \leq f\}$ have compact components,

Received by the editors September 16, 1986 and, in revised form, November 6, 1986. 1980 Mathematics Subject Classification (1985 Revision). Primary 26A21; Secondary 54C08. Key words and phrases. Darboux, connectivity, Baire's first class. 
PR: $\quad f: I \rightarrow R$ is said to have a perfect road at each point if for every $x \in I$, there exists a perfect set $P$, having $x$ as a bilateral limit point such that $f \mid P$ is continuous at $x$,

Zc: $\quad f: I \rightarrow R$ satisfies Zahorski's condition, Zc, if each set $\{f<a\}$ and $\{f>a\}$ is bilaterally $c$-dense in itself,

$\mathrm{Z} \omega: \quad f: I \rightarrow R$ satisfies condition $\mathrm{Z} \omega$, if each set $\{f<a\}$ and $\{f>a\}$ is bilaterally dense in itself.

The usual conventions are adopted at the endpoints of $I$ when some condition is supposed to hold bilaterally, as well as conventions about cases where sets such as $\{f \leq a\}$ or $\{f>a\}$ might be empty. Now, consider two more topological function properties:

AC: $\quad f: X \rightarrow Y$ is said to be almost continuous (in the sense of Stallings [18]) if for every open set $G \subseteq X \times Y$ containing $f$, there exists a continuous $g: X \rightarrow Y$ lying entirely in $G$.

EXT: $\quad f: X \rightarrow Y$ is said to be extendable provided there exists a connectivity function $g: X \times I \rightarrow Y$ such that $f(x)=g(x, 0)$ for each $x \in X$.

Stallings showed in [18] that for functions $f: I \rightarrow R, \mathrm{AC} \Rightarrow$ Conn, but that for functions $f: I^{2} \rightarrow R$, Conn $\Rightarrow$ AC. From this it follows that for functions $f: I \rightarrow R, \mathrm{EXT} \Rightarrow \mathrm{AC} \Rightarrow \mathrm{Conn}$, and Stallings asked if the implications were reversible. It was shown in $[\mathbf{4}, \mathbf{1 1}$, and 17] that $\mathrm{AC} \notin$ Conn, and it was recently shown by Gibson and Roush [7] that EXT $\nLeftarrow$ AC in the case of real functions. It was shown in [1] that $\mathrm{AC} \Leftrightarrow$ Conn within the class $\mathrm{B}_{1}$, and it will be shown below that property EXT is also equivalent to all of these other topological properties within this class.

It is the purpose of this paper to investigate how these ten topological properties are related to each other within the following classes of functions:

$\mathrm{F}=$ the class of all functions from $I$ into $R$.

$\mathrm{L}=$ the class of Lebesgue measurable functions in $F$.

$\mathrm{B}=$ the class of Borel measurable functions in $F$.

$\mathrm{J}_{1}=$ the class of pointwise limits of sequences of functions in $F$ which have only discontinuities of the first kind.

$\mathrm{R}_{1}=$ the class of pointwise limits of sequences of functions in $F$ which are continuous from the right.

$\mathrm{B}_{1}=$ the class of pointwise limits of sequences of continuous functions in $F$.

It follows from the work of Reed [16] that

$$
\mathrm{B}_{1} \Rightarrow \mathrm{R}_{1} \Rightarrow \mathrm{J}_{1} \Rightarrow \mathrm{B} \Rightarrow \mathrm{L} \Rightarrow \mathrm{F}
$$

and that the implications are irreversible.

The theorems and examples given in $\S$ II and III represent an expansion of the results in an earlier paper [1], where the relationships between properties AC, Conn, $D$, and $P C$ within the classes $B_{1}, R_{1}$, and $J_{1}$ were investigated. We do not completely explain all the relationships in that we have as yet been unable to determine whether EXT $\Leftarrow A C$ within the classes $B, J_{1}$, or $R_{1}$. As a major step in showing EXT $\Leftarrow \mathrm{DB}_{1}$, we establish a theorem about metric density which we hope will have other applications. 


\section{Theorems and examples.}

THEOREM 1. Within F, only the following implications hold

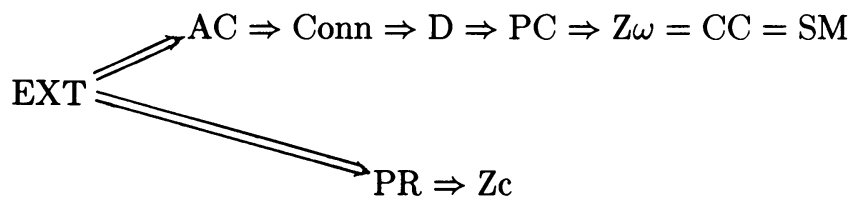

and the diagram is the same within $\mathrm{L}$.

PROOF. Proofs of most of the implications are straightforward and have appeared elsewhere. That EXT $\Rightarrow \mathrm{AC} \Rightarrow$ Conn follows from Stalling's work [18]. The implication EXT $\Rightarrow$ PR has recently been shown by Gibson and Roush [8].

To show that the diagram of implications does not change within $\mathrm{L}$, we would need to describe Lebesgue measurable examples where $\mathrm{AC} \nRightarrow \mathrm{PR}, \mathrm{AC} \notin \mathrm{Conn}$, Conn $\nLeftarrow \mathrm{D}, \mathrm{D} \nLeftarrow \mathrm{PR}, \mathrm{PC} \nRightarrow \mathrm{Zc}$, and $\mathrm{PC} \nLeftarrow \mathrm{Zc}$. We will give only the first example here. The other examples are discussed below, and are better than just Lebesgue measurable.

The first example given by Gibson and Rousch in [6] shows that $\mathrm{AC} \nRightarrow \mathrm{PR}$, but that example is not Lebesgue measurable. Therefore, we modify that example as follows. Let $C$ be the ternary Cantor set, and Let $C^{0}=C-\{$ endpoints of $C\}$. Construct $f$ so that (1) $f\left[I-C^{0}\right]=\{0\}$ and (2) $f$ intersects every closed subset of $C \times I$ which has an uncountable $x$-projection. Then $f$ is not PR because of the points $x \in C^{0}$ for which $f(x)>0$. To see that $f$ is AC, let $G$ be an open subset of $I \times R$ such that $f \subseteq G$. $(C \times I) \backslash G$ is closed and its $x$-projection $P$ must be countable. Let $P=\left\{x_{1}, x_{2}, \ldots\right\}$. For each $i$, put a box $\left(a_{i}, b_{i}\right) \times\left(c_{i}, d_{i}\right)$ around $\left(x_{i}, f\left(x_{i}\right)\right)$ whose closure is inside $G$ such that $a_{i}$ and $b_{i}$ are not in $C$. Some finite subcollection $\left\{\left(a^{1}, b^{1}\right),\left(a^{2}, b^{2}\right), \ldots,\left(a^{n}, b^{n}\right)\right\}$ of $\left\{\left(a_{1}, b_{1}\right),\left(a_{2}, b_{2}\right), \ldots\right\}$ covers $P$. For each $i$, let $\left(c^{i}, d^{i}\right)$ be the appropriate $\left(c_{j}, d_{j}\right)$ that originally went with $\left(a^{i}, b^{i}\right)$. We will build a continuous function $g_{i}$ with domain $\left[a^{i}, b^{i}\right]$ and which lies in $G$. Then $a^{i}$ and $b^{i}$ are not in $P$ and $P$ is closed. Since $\left(a^{i}, b^{i}\right) \cap P$ is not empty, we let $e^{i}=\inf \left\{\left(a^{i}, b^{i}\right) \cap P\right\}$ and $f^{i}=\sup \left\{\left(a^{i}, b^{i}\right) \cap P\right\}$. If $e^{i}$ is not a left endpoint of $C$, there is an $x$ between $a^{i}$ and $e^{i}$ such that $\{x\} \times I$ lies in $G$. If $e^{i}$ is a left endpoint of $C$, there will be an $x$ between $e^{i}$ and $f^{i}$ such that $\left[e^{i}, x\right] \times\{0\}$ and $\{x\} \times I$ lie in $G$. In either case, it will be possible to construct a polygonal line graph which is continued in $G$, extends horizontally from $\left(a^{i}, 0\right)$ to a point just to the left of $(x, 0)$, and then slants up with positive slope to a point inside $\left(a^{i}, b^{i}\right)$ × $\left(c^{i}, d^{i}\right)$ with abscissa just greater than $x$. We then do a similar thing, moving from $\left(b^{i}, 0\right)$ to the left. Connecting the two polygons within $\left(a^{i}, b^{i}\right) \times\left(c^{i}, d^{i}\right)$ completes the definition of $g_{i}$.

Now, extend each $g_{i}$ to $[0,1]$ so that $g_{i}(x)=0$ outside $\left[a^{i}, b^{i}\right]$. Then, $g=$ $\sup \left\{g_{1}, g_{2}, \ldots, g_{n}\right\}$ is a continuous function with domain [0,1], lying in $G$.

THEOREM 2. Within B, only the following implications hold:

$$
\mathrm{EXT} \Rightarrow \mathrm{AC} \Rightarrow \mathrm{Conn} \Rightarrow \mathrm{D} \Rightarrow \mathrm{PR} \Rightarrow \mathrm{PC} \Rightarrow \mathrm{Z} \omega=\mathrm{CC}=\mathrm{SM}
$$


with the possible exception $\mathrm{EXT} \Leftarrow \mathrm{AC}$, none of the remaining implications is reversible. The same is true within the class $\mathrm{J}_{1}$.

ProOF. The improvement in the diagram of implications is that we have $\mathrm{D} \Rightarrow$ PR in B. The proof that Conn $\Rightarrow$ PR given on p. 10 of [3] really uses only that $f$ is Borel, rather than $B_{1}$ (as is pointed out on p. 11). A slight modification of that proof yields the fact that $\mathrm{DB} \Rightarrow \mathrm{PR}$.

To show that the implications not involving property EXT do not improve in $\mathrm{J}_{1}$ consider the following examples.

The characteristic function of the irrationals shows that $\mathrm{J}_{1} \mathrm{PC} \nRightarrow \mathrm{Zc}$.

The characteristic function for $C^{0}$ shows that $\mathrm{J}_{1} \mathrm{PR} \nRightarrow \mathrm{D}$, and if this function is altered at one point of $C^{0}$ to have value $1 / 2$, the resulting function shows that $\mathrm{J}_{1} \mathrm{Zc} \nRightarrow \mathrm{PC}$.

Examples which show that $\mathrm{J}_{1} \mathrm{D} \nRightarrow \mathrm{Conn}$ and that $\mathrm{R}_{1}$ Conn $\nRightarrow \mathrm{AC}$ were given in [1].

THEOREM 3. Within $\mathrm{R}_{1}$, the following implications hold:

$$
\mathrm{EXT} \Rightarrow \mathrm{AC} \Rightarrow \mathrm{Conn}=\mathrm{D}=\mathrm{PR}=\mathrm{PC}=\mathrm{Zc}=\mathrm{Z} \omega=\mathrm{CC}=\mathrm{SM}
$$

and the second implication is irreversible.

Proof. The only new implication, that Conn $\Leftarrow D$ in $R_{1}$, is proved in $[\mathbf{1}]$, where the example to show $R_{1}$ Conn $\nRightarrow A C$ is also given.

III. Metric density and the proof that $\mathrm{DB}_{1} \Rightarrow \mathrm{EXT}$. As a primary tool in showing that $\mathrm{DB}_{1} \Rightarrow \mathrm{EXT}$, we prove a theorem about metric density in $\mathbf{R}^{n}$. This theorem utilizes the notion of the Besicovich covering constant for dimension $n$ described in the following theorem. $\lambda_{n}$ denotes Lebesgue measure in $\mathbf{R}^{n}$, and $B(x, \delta)$ denotes the ball with center $x$ and radius $\delta$. A function $f: R \rightarrow R$ is approximately continuous if for every $x \in R$ and every $\varepsilon>0, x$ is a point of density of $\{y:|f(x)-f(y)|<\varepsilon\}$.

THEOREM B. For every dimension $n$ there is a positive constant $K_{n}$ with the following property: For every positive function $\delta$ defined on a bounded measurable set $A \subset \mathbf{R}^{n}$, there is a finite collection of mutually disjoint balls $\left\{B\left(x_{i}, \delta_{x_{i}}\right): i=\right.$ $1,2, \ldots, m\}$ for which

$$
\lambda_{n}\left(\bigcup_{i=1}^{m} B\left(x_{i}, \delta_{x_{i}}\right) \cap A\right)>\lambda_{n}(A) / K_{n} .
$$

ProOF. Theorem B is contained in Theorem 2.8.14 of [5]. This is because the Euclidean metric on $\mathbf{R}^{n}$ is "directionally $\xi, \eta, \varsigma$ limited" on all of $\mathbf{R}^{n}$ with $\xi=+\infty$, $\eta=1 / 3$, and $\varsigma$ chosen suitably to work uniformly on all of $\mathbf{R}^{n}$ (for $n=1, \varsigma=2$ and for $n=2, \varsigma=6$ ). Then our $K_{n}=2 \varsigma+1$.

THEOREM 4. Let $x_{0}$ be a density point of the measurable set $H \subseteq \mathbf{R}^{n}$, let $\varepsilon>0$ be fixed, and let

$$
D_{\delta}=\left\{x: \frac{\lambda_{n}(B(x, h) \cap H)}{\lambda_{n}(B(x, h))}>1-\varepsilon \text { for every } 0<h<\delta\right\} \quad(\delta>0) .
$$


Then

$$
\operatorname{limit}_{(t, \delta) \rightarrow(0,0)} \frac{\lambda_{n}\left(B\left(x_{0}, t\right) \cap D_{\delta}\right)}{\left.\lambda_{n} B\left(x_{0}, t\right)\right)}=1 .
$$

ProOF. Let $0<\eta<1$ be given and let $\delta_{0}>0$ be such that

$$
\frac{\lambda_{n}(B \cap H)}{\lambda_{n}(B)}>1-\frac{\eta}{K_{n} 2^{n}} \varepsilon
$$

for every ball $B$ containing $x_{0}$ and diameter $<\delta_{0}$. Here $K_{n}$ is the Besicovich covering constant for dimension $n$. We now prove that if $0<t$ and $\delta<\delta_{0} / 4$ then

$$
\frac{\lambda_{n}\left(B\left(x_{0}, t\right) \cap D_{\delta}\right)}{\lambda_{n}\left(B\left(x_{0}, t\right)\right)}>1-\eta
$$

Suppose this is not true and let $t, \delta \in\left(0, \delta_{0} / 4\right)$ be fixed with

$$
\lambda_{n}\left(B\left(x_{0}, t\right) \cap D_{\delta}\right) \leq(1-\eta) \lambda_{n}\left(B\left(x_{0}, t\right)\right) .
$$

Let $A=B\left(x_{0}, t\right) \backslash D_{\delta}$. Then clearly $\lambda_{n}(A) \geq \eta \lambda_{n}\left(B\left(x_{0}, t\right)\right)$ and for each $x \in A$ there is an $h_{x} \in(0, \delta)$ with

$$
\lambda_{n}\left(B\left(x, h_{x}\right) \cap H\right) \leq(1-\varepsilon) \lambda_{n}\left(B\left(x, h_{n}\right)\right) .
$$

Hence by Theorem $\mathrm{B}$, there is a disjoint family $\left\{B\left(x_{i}, h_{x_{i}}\right): i=1,2, \ldots, m\right\}$ such that

$$
\lambda_{n}(U) \geq \lambda_{n}(A \cap U)>\lambda_{n}(A) / K_{n} \quad \text { where } U=\bigcup_{i=1}^{m} B\left(x_{i}, h_{x_{i}}\right) .
$$

Obviously, then, $\lambda_{n}(U \cap H) \leq(1-\varepsilon) \lambda_{n}(U)$. First we verify that $h_{x_{i}}<t$ for every $i=1,2, \ldots, m$. Indeed, if $h_{x_{i}} \geq t$ for some $i$ then $x_{0} \in B\left(x_{i}, h_{x_{i}}\right) \equiv B$, and $\operatorname{diam}(B)<2 \delta<\delta_{0}$. But then,

$$
\lambda_{n}(B \cap H)>\left(1-\frac{\eta}{K_{n} 2^{n}} \varepsilon\right) \lambda_{n}(B)>(1-\varepsilon) \lambda_{n}(B)
$$

which contradicts the choice of $h_{x_{i}}$. Hence $h_{x_{i}}<t$ for every $i=1,2, \ldots, m$ and as a consequence, $U \subseteq B\left(x_{0}, 2 t\right)$. Then,

$$
\begin{aligned}
\lambda_{n}\left(B\left(x_{0}, 2 t\right) \cap H\right) & \leq \lambda_{n}(H \cap U)+\lambda_{n}\left(B\left(x_{0}, 2 t\right) \backslash U\right) \\
& \leq(1-\varepsilon) \lambda_{n}(U)+\lambda_{n}\left(B\left(x_{0}, 2 t\right)\right)-\lambda_{n}(U) \\
& =\lambda_{n}\left(B\left(x_{0}, 2 t\right)\right)-\varepsilon \lambda_{n}(U) \leq \lambda_{n}\left(B\left(x_{0}, 2 t\right)\right)-\frac{\varepsilon}{K_{n}} \lambda_{n}(A) \\
& \leq \lambda_{n}\left(B\left(x_{0}, 2 t\right)\right)-\frac{\varepsilon \eta}{K_{n}} \lambda_{n}\left(B\left(x_{0}, t\right)\right) \\
& =\lambda_{n}\left(B\left(x_{0}, 2 t\right)\right)\left(1-\frac{\varepsilon \eta}{K_{n} 2^{n}}\right)
\end{aligned}
$$

Since $t<\delta_{0} / 4$, this entails a contradiction.

COROLlaRY. Let $x_{0}$ be a density point of the measurable set $H \subseteq \mathbf{R}^{n}$, let $\varepsilon>0$ be fixed and let

$$
D_{\delta}=\left\{x: \frac{\lambda_{n}(B(x, h) \cap H)}{\lambda_{n}(B(x, h))}>1-\varepsilon \text { for every } 0<h<\delta\right\} \quad(\delta>0) .
$$


Then there is an $\eta>0$ such that

$$
\lambda_{n}\left(B\left(x_{0}, t\right) \cap D_{\delta}\right)>\lambda_{n}\left(B\left(x_{0}, t\right)\right) / 2 \text { for every } 0<t, \delta \leq \eta .
$$

THEOREM 5. Let $f: \mathbf{R} \rightarrow \mathbf{R}$ be bounded and approximately continuous and let

$$
F(x, h)= \begin{cases}\frac{1}{2 h} \int_{x-h}^{x+h} f(t) d t & \text { if } h \neq 0, \\ f(x) & \text { if } h=0 .\end{cases}
$$

Then, $F$ is $\mathrm{PC}$ on $\mathbf{R}^{2}$. (Note that $\mathrm{PC}=$ Conn for functions $\left.f: \mathbf{R}^{2} \rightarrow \mathbf{R}[\mathbf{9}, \mathbf{1 0}].\right)$

PROOF. The function $F$ is continuous at each point $(x, h)$ with $h \neq 0$ so that it suffices to show that $F$ is peripherally continuous at $\left(x_{0}, 0\right)$ for every $x_{0} \in \mathbf{R}$. Let $\varepsilon>0$ be given. We show that there is a rectangle $R=[a, b] \times[c, d]$ such that $x_{0}-\varepsilon<$ $a<x_{0}<b<x_{0}+\varepsilon$ and $-\varepsilon<c<0<d<\varepsilon$ for which $\left|F(x, h)-F\left(x_{0}, 0\right)\right|<\varepsilon$ for every point $(x, h)$ on the boundary of $R$. Let $H=\left\{x:\left|f(x)-f\left(x_{0}\right)\right|<\varepsilon / 2\right\}$; then $x_{0}$ is a point of density of $H$ and we define

$$
D_{\delta}=\{x: \lambda((x-h, x+h) \cap H)>2 h(1-\varepsilon / 2) \text { for } 0<h \leq \delta\} .
$$

Using the Corollary, there is $0<\eta<\varepsilon$ such that $\lambda\left(\left(x_{0}-t, x_{0}+t\right) \cap D_{\delta}\right)>t$ for every $0<t, \delta<\eta$. This implies that $x_{0}$ is a bilateral limit point of $D_{\delta}$. Since $f$ is bounded and approximately continuous at $x_{0}$,

$$
\operatorname{limit}_{h \rightarrow 0} F\left(x_{0}, h\right)=f\left(x_{0}\right) \text {. }
$$

Let $-\eta<c<0<d<\eta$ be such that both $\left|F\left(x_{0}, c\right)-f\left(x_{0}\right)\right|<\varepsilon$ and $\mid F\left(x_{0}, d\right)-$ $f\left(x_{0}\right) \mid<\varepsilon$. By the continuity of $F$ there is $0<\delta<\varepsilon$ such that both $\mid F(x, c)-$ $f\left(x_{0}\right) \mid<\varepsilon$ and $\left|F(x, d)-f\left(x_{0}\right)\right|<\varepsilon$ whenever $x \in\left(x_{0}-\delta, x_{0}+\delta\right)$. Let $a \in$ $\left(x_{0}-\delta, x_{0}\right) \cap D_{\eta}$ and $b \in\left(x_{0}, x_{0}+\delta\right) \cap D_{\eta}$. Then for every $|h|<\eta$,

$$
\lambda((x-h, x+h) \cap H)>2 h(1-\varepsilon / 2) \text { for } x=a \text { or } b .
$$

Supposing, as we may, that $|f| \leq 1$, this implies that for $x=a$ or $b$ and $|h|<\eta$,

$$
\begin{aligned}
& \left|\frac{1}{2 h} \int_{x-h}^{x+h} f(t) d t-f\left(x_{0}\right)\right| \leq \frac{1}{2 h} \int_{x-h}^{x+h}\left|f(t)-f\left(x_{0}\right)\right| d t \\
& \quad=\frac{1}{2 h}\left[\int_{[x-h, x+h] \cap H}\left|f(t)-f\left(x_{0}\right)\right| d t+\int_{[x-h, x+h] \backslash H}\left|f(t)-f\left(x_{0}\right)\right| d t\right] \\
& \quad<\frac{\varepsilon}{2}+\frac{\varepsilon}{2}=\varepsilon .
\end{aligned}
$$

this shows that $R=[a, b] \times[c, d]$ has the desired properties and the proof is complete.

THEOREM 6. Within $\mathrm{B}_{1}$, the following is true:

$$
\mathrm{EXT}=\mathrm{AC}=\mathrm{Conn}=\mathrm{D}=\mathrm{PR}=\mathrm{Zc}=\mathrm{PC}=\mathrm{Z} \omega=\mathrm{CC}=\mathrm{SM} .
$$

PROOF. We will show that $\mathrm{DB}_{1} \Rightarrow \mathrm{EXT}$ by reducing the problem to the bounded approximately continuous case and calling on Theorem 5 . Let $f$ be $\mathrm{DB}_{1}$. It follows from "Maximoff's Theorem" [14] (see p. 36 of [3]) that there exists a homeomorphism $h$ of $I$ onto itself such that $f \circ h$ is approximately continuous (actually, there was some problem associated with the original proof of this theorem, 
but this has recently been straightened out by Preiss in [15]). Then, let $A$ be the arctangent function and $g=A \circ(f \circ h) . g$ is bounded and approximately continuous. Now, assume $g$ is extended appropriately to $\mathbf{R}$ and define

$$
G(x, k)= \begin{cases}\frac{1}{2 k} \int_{x-k}^{x+k} g(t) d t & \text { if } k \neq 0, \\ f(x) & \text { if } k=0\end{cases}
$$

as in Theorem 5. $G$ is PC (and therefore Conn). Since $g(t)$ is approximately continuous and $-\pi / 2<g(t)<\pi / 2$ for every $t$, it follows that $-\pi / 2<G(x, k)<\pi / 2$ for every $x$ and $k$. Thus we can define $\mathrm{F}$ to be $F(x, k)=A^{-1}\left[G\left(h^{-1}(x), k\right)\right]$ for each $x$ and $k$. F is still Conn and is an extension of the original $f$. This completes the proof.

We now state several open problems.

Problem 1. EXT $\nLeftarrow A C$ in $\mathrm{L}$ but EXT $\Leftarrow A C$ in $\mathrm{B}_{1}$. What is the first class among $\mathrm{B}, \mathrm{J}_{1}, \mathrm{R}_{1}$ in which $\mathrm{AC} \Rightarrow \mathrm{EXT}$ ?

Problem 2. How are the topological properties related within the function classes:

$\mathrm{U}$ : universally measurable functions,

$B_{w}$ : functions with the Baire property in wide sense,

$B_{r}$ : functions with the Baire property in restricted sense,

(s): Marczewski-measurable functions?

These classes fit with the others as follows:

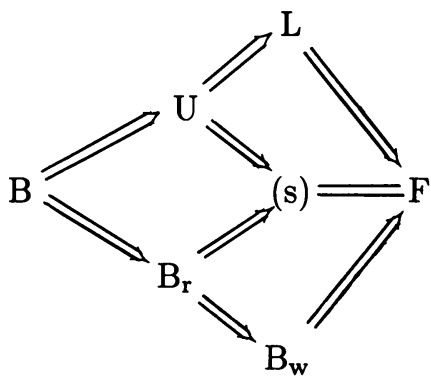

See [2] for definitions and discussion.

Problem 3. Extend this investigation to higher dimensions. Certainly, the classes $B_{1}, R_{1}, B$ have natural analogs for functions of two real variables, as do properties EXT, AC, Conn, D, PR (without bilateralness), PC, Zc and $\mathrm{Z} \omega$ (without bilateralness), and CC.

\section{REFERENCES}

1. J. B. Brown, Almost continuous Darboux functions and Reed's pointwise convergence criteria, Fund. Math. 86 (1974), 1-7.

2. J. B. Brown and K. Prikry, Variations on Lusin's theorem, Trans. Amer. Math. Soc. 302 (1987), 77-86.

3. A. M. Bruckner, Differentiation of real functions, Lecture Notes in Math., vol. 659, SpringerVerlag, Berlin, Heidelberg and New York, 1978.

4. J. L. Cornette, Connectivity functions and images on Peano continuua, Fund. Math. 58 (1966), 183-192. 
5. H. Federer, Geometric measure theory, Springer-Verlag, New York, 1969.

6. R. Gibson and F. Roush, The Cantor intermediate value property, Topology Proc. 7 (1982), 55-62.

7. _ Concerning the extension of connectivity functions, Topology Proc. (to appear).

8. Connectivity functions with a perfect road, Real Anal. Exchange 11 (1985-86), 260-264.

9. M. R. Hagan, Equivalence of connectivity maps and peripherally continuous transformations, Proc. Amer. Math. Soc. 17 (1966), 175-177.

10. O. H. Hamilton, Fixed points for certain noncontinuous transformations, Proc. Amer. Math. Soc. 8 (1957), 750-756.

11. F. B. Jones and E. S. Thomas, Jr., Connected $G_{\delta}$-graphs, Duke Math. J. 33 (1966), 341-345.

12. B. Knaster and C. Kuratowski, Sur quelques proprietes topologiques des fonctions derivees, Rend. Circ. Mat. Palermo 49 (1925), 382-386.

13. C. Kuratowski and W. Sierpinski, Les fonctions de classe I et les ensembles connexes punctiformes, Fund. Math. 3 (1922), 303-313.

14. I. Maximoff, Sur la transformation continue de fonctions, Bull. Soc. Phys. Math. Kazan (3) 12 (1940), 9-41.

15. D. Preiss, Maximoff's theorem, Real Anal. Exchange 5 (1979-80), 92-104.

16. C. S. Reed, Pointwise limits of sequences of functions, Fund. Math. 67 (1970), 183-193.

17. J. H. Roberts, Zero-dimensional sets blocking connectivity functions, Fund. Math. 57 (1965), 173-179.

18. J. Stallings, Fixed point theorems for connectivity maps, Fund. Math. 47 (1959), 249-263.

Department of Mathematics, Auburn University, Auburn, Alabama 36849

Department of mathematics, St. Olaf College, Northfield, Minnesota 55057

DEPARTMENT OF ANALYSIS, EÖTVÖS LORÁND UNIVERSITY, BUDAPEST, HUNGARY 